\title{
A psychiatric perspective view of bariatric surgery patients
}

\author{
Isabel Brandão1,2, Ana Luísa Fernandes³, Eva Osório1,2, Maria da Conceição Calhaư4, Rui Coelho1,2 \\ ${ }^{1}$ Department of Clinical Neurosciences and Mental Health, Faculty of Medicine, University of Oporto, Oporto, Portugal. \\ 2 Department of Psychiatry, Centro Hospitalar de S. João, Oporto, Portugal. \\ 3 Faculty of Medicine, University of Oporto, Oporto, Portugal. \\ ${ }^{4}$ Department of Biochemistry, Faculty of Medicine, University of Oporto, Oporto, Portugal.
}

Received: 4/18/2015 - Accepted: 10/26/2015

DOI: 10.1590/0101-60830000000062

\begin{abstract}
Background: Bariatric surgery is the only procedure that has significant results in weight loss and improvements in medical comorbidities in morbid obese patients. Severely obese patients are also associated with a higher prevalence of psychiatric disorders and poor quality of life. Objective: To evaluate specific areas of psychopathology in individuals undergoing bariatric surgery. Methods: A review of the literature was conducted from January 2002 to March 2014 by researching PubMed database using the following query: "morbid AND obesity AND bariatric AND surgery AND (psychiatry OR psychology)". Results: Overall improvements in eating behaviors, mood disorders and body image are reported after bariatric surgery, and the mechanism is not enlightened. Risk of suicide and consumption of substances of abuse, especially alcohol, after gastric bypass surgery are problems that clinicians must be aware. Discussion: Bariatric patients should be monitored after surgery to identify who did not show the expected benefits postoperatively and the ones who develop psychiatric symptoms after an initial positive response.
\end{abstract}

Brandão I et al. / Arch Clin Psychiatry. 2015;42(5):122-8

Keywords: Morbid obesity, bariatric surgery, psychiatry, psychology.

\section{Introduction}

Obesity is associated with increased risk of medical complications ${ }^{1}$. Morbid obesity (MO) is defined as having a body mass index (BMI) $\geq 40 \mathrm{~kg} / \mathrm{m}^{2}$. In the United States, between 2000 and 2010, the prevalence of a BMI $>40 \mathrm{~kg} / \mathrm{m}^{2}$ increased by $70 \%$, whereas the prevalence of BMI $>50 \mathrm{~kg} / \mathrm{m}^{2}$ increased even faster ${ }^{2}$.

Pharmacological treatment, diet regimens and lifestyle alterations do not seem to be effective in morbidly obese patients ${ }^{3}$. Therefore, bariatric surgery is considered the most effective treatment in severe obesity, yielding more enduring weight loss ${ }^{1}$. Several surgical procedures are performed: Roux-en-Y gastric bypass (RYGB), sleeve gastrectomy, laparoscopic adjustable gastric banding (LAGB), vertical banded gastroplasty and biliopancreatic diversion ${ }^{1,3,4}$.

Psychiatric problems are also common among morbidly obese patients and bariatric surgery may often contribute to improve it. The Swedish obese subjects (SOS) intervention study compared several psychosocial variables between a surgical group and a conventional group treated with diet and exercise counseling over ten years of follow-up. A significantly better outcome in depression, health perception, social interaction and psychosocial functioning was achieved by the surgical group whereas no significant differences were found in overall anxiety ${ }^{5}$. Furthermore, patients submitted to surgery lose significantly more weight than non-operated patients ${ }^{6}$.

Several studies have reported a higher prevalence of psychiatric disorders in patients seeking bariatric surgery comparing to general population 7 , as seen in other medical diseases, such as cancer ${ }^{8,9}$. Preoperative studies demonstrated that 20\%-56\% had a current psychiatric diagnosis ${ }^{10}$. Furthermore, at least one Axis I disorder was reported by $37 \%-43 \%$ of patients ${ }^{11,12}$. Recently, one study stated social phobia as the most prevalent Axis I disorder ${ }^{11}$, in contrast to previous studies, where major depressive disorder was the most common $10,12,13$. In addition, studies reported preoperative prevalence rates of $15 \%-33 \%$ for mood disorders and of $24 \%-30 \%$ for anxiety disorders ${ }^{10-12}$. High incidences of somatization, hypochondria and obsessive compulsive disorder were also found in bariatric patients ${ }^{14}$. Moreover, the prevalence of binge eating behaviors and binge eating disorder (BED) is variable with a range from $4 \%-40 \%^{11,15-18}$.
A large number of published studies have shown an overall improvement in mental health and psychosocial factors related to metabolic improvement and weight loss in most individuals undergoing weight loss surgery ${ }^{19,20}$. Additionally, a greater appreciation of life, greater sense of inner strength and improvement of interpersonal capabilities were experienced ${ }^{21}$. This alleviation of psychological, psychosocial and psychiatric status led to the suggestion that the negative psychological features result from the condition, the patient's severe obesity, and not the underlying character of the individual. On the other hand, improvements in psychological functioning were observed even though some patients remained obese after surgery when only a small amount of weight was lost. Therefore, greater optimism, higher self-esteem and taking an active role in life, leads to a better outcome ${ }^{20}$.

However, not all studies have demonstrated a positive impact of bariatric surgery and a growing body of evidence has suggested that some of the stated benefits seem to be limited to the first years following weight loss surgery ${ }^{22}$. For instance, Scholtz et al. reported that nearly half of the sample developed a psychiatric disorder five years after bariatric surgery and those with a past of a psychiatric disturbance being significantly more at risk. Furthermore, postoperative psychiatric disorder predicted a lower probability of achieving a good outcome ${ }^{23}$.

It has been reported that despite limited weight loss or side effects following bariatric surgery, most patients were satisfied with the results and did not regret the surgical option ${ }^{19}$. However, up to $20 \%$ appeared to be dissatisfied and the reasons pointed were psychosocial problems, surgical complications and not fulfilling their weight expectations $s^{3,7}$.

Therefore, weight loss and improvement in medical comorbidities should be seen as successful factors after bariatric surgery, but the amelioration of psychiatric symptoms, eating behavior, psychosocial variables and quality of life are important outcomes to consider ${ }^{3}$. Thus, the aim of this review is to evaluate specific areas of psychopathology in individuals with morbid obesity underwent to bariatric surgery, such as, eating behaviors, mood disorders, body image, suicide and substances of abuse. 
This is relevant because psychological aspects potentially due to the postoperatively dramatic effects observed in weight loss, metabolic and behavior changes may be easily forgotten.

\section{Methods}

The relevant literature was identified by searching in PubMed database. Some limits were established prior to be searched, such as, publication date from January of 2002 to June of 2015, studies in humans and articles written in English.

The query used was: "morbid" AND "obesity" AND "bariatric" AND "surgery" AND ("psychiatry" OR "psychology"). A total of 422 articles were found. After eliminating the duplicates, 416 articles remained.

From the initial group of articles identified, 286 were excluded after abstract reading phase. The following exclusion criteria were used: studies in children or adolescents $(n=33)$; preoperative studies $(n=88)$; evaluating other aspects postoperatively that not psychiatric symptoms or disorders $(n=48)$; specific population studies $(n=7)$; non-morbid obese patients $(n=2)$; description of surgical procedures $(n=14)$; studies for analyze of psychological questionnaires $(n=15)$; studies related to medical comorbidities $(n=25)$; studies related to plastic surgery $(n=15)$; non-surgical studies $(n=13)$; studies with no available abstract $(n=12)$; clinical cases or opinion pieces $(n=14)$.

From the 130 articles of interest, the full-text of 20 articles remained inaccessible and 110 full-texts were read. The following inclusion criteria were used: studies which evaluated post-operative eating behaviors, post-operative weight loss, mood disorders, suicide, body image and substance use. Therefore, a total of 75 articles were included from the research. Further articles were included after inspection of references list from relevant articles.

\section{Results}

\section{Eating behaviors}

Eating patterns such as binge eating, night eating syndrome, "grazing" and excessive fluid intake are common in bariatric seeking population ${ }^{24}$.

Assessing postsurgical data regarding eating behavior is considered difficult and one of the pointed drawbacks is the difference in definitions of binge eating employed by different studies ${ }^{18}$. Binge eating disorder (BED) is characterized by recurring episodes of eating significantly more food in a short period of time than most people would eat under similar circumstances, with episodes marked by feelings of lack of control. It is associated with marked distress, physical and psychological problems and occurs, on average, at least once a week every three months ${ }^{25}$.

Despite the inconsistencies, the majority of studies report a decrease in binge eating and an improvement in eating behavior after bariatric surgery $y^{4,26,27}$. Moreover, most patients report a smaller amount of food eaten, a decrease in hunger, an earlier satiety, less inadequate eating behaviors and more control over their food intake ${ }^{22,27}$. When patients have binge eating behaviors postoperatively, it is being associated with reduced mental health and poor weight outcome ${ }^{28-32}$.

Several studies have evaluated differences in weight and psychopathology between binge eaters (BE) and non-binge eaters (NBE) postoperatively. We need to be aware which surgery procedure was selected and we must account with that because we can have only a decrease of the stomach size or a reduction of the amount of calories absorbed by bypassing portions of the intestine also. A study compared psychopathological differences between BE and NBE after vertical banded gastroplasty in which the $\mathrm{BE}$ group reported higher scores for symptoms of eating disturbances, depression, alcohol dependence and personality disorders than NBE. Furthermore, the $\mathrm{BE}$ group lost less weight than the non-binge eating ${ }^{33}$. In other study in patients undergoing Roux-en-Y gastric bypass the authors found a less successful outcome for the BE patients compared with NBE in psychological wellbeing and quality of life ${ }^{34}$.
Although the ingestion of large amounts of food becomes nearly impossible after gastrointestinal changes due to bariatric surgery, several maladaptive eating behaviors have been reported postoperatively. The frequency of these behaviors help to predict poorer weight loss, weight regain ${ }^{28}$ and postoperative complications ${ }^{35}$, such as inability to control food urges, decreased well-being, concerns over addictive behaviors (alcohol and substance abuse), lack of self-monitoring and fewer postoperative follow-up visits ${ }^{36}$. Several studies have demonstrated that a subgroup of patients after surgery will develop subjective binge or "loss of control" over eating ${ }^{37,38}$. "Grazing" is characterized by continual ingestion of small amounts of food over extended time with feelings of loss of control and some authors have suggested that it is a manifestation of binge eating behavior postoperatively ${ }^{15,39}$. According to Zunker et al., bariatric surgery patients seem to interpret "grazing" as a healthy eating behavior characterized by mindful food choices that are consumed in small amounts frequently throughout the day ${ }^{39}$. However, the result is a greater intake of food which often ends in weight regain ${ }^{20,40,41}$.

Emotional/stress eaters have difficulty in dealing with negative emotions and food may provide a transient shelter to avoid confronting difficult feelings ${ }^{42}$. The type of food usually consumed during these episodes, such as, candies or fast food have negative gastrointestinal symptoms, for example, nausea, vomiting or dumping syndrome. However, some patients persist in their attempts to eat in response to stress or emotional events. Instead of the large amount they used to ingest before surgery, patients consume high-fat and sugar content food in smaller amounts or drink sugar-sweetened beverages, so they will experience minimal gastrointestinal adverse symptoms ${ }^{43}$. Snacking post-surgery has been associated with poor weight loss ${ }^{35,44}$.

Night eating syndrome (NES) is characterized by recurrent episodes of evening hyperphagia, poor sleep onset or maintenance, morning anorexia and a habit of getting up to eat during nocturnal awakenings. Additionally, the night eating has to be associated with significant distress ${ }^{45}$. A few studies have investigated this disorder after bariatric surgery. Latner et al. have reported that postoperative nocturnal eating is associated with greater postsurgical BMI and less treatment satisfaction ${ }^{46}$. On the other hand, Morrow et al. have shown no differences in weight loss after RYBG between the night eaters group and control group ${ }^{47}$. However, diagnostic criteria are inconsistent between studies which make it difficult to establish comparisons and draw conclusions. Therefore, NES definition should be revised and an agreement on the behavioral features and diagnostic criteria should be established. Furthermore, more studies evaluating this disorder in the severely obese population and the impact on bariatric surgery outcomes are required.

Symptoms such as vomiting, constipation and decreasing appetite are often seen after bariatric surgery. However, these symptoms may also be reported by patients with eating disorders. Therefore, it may be difficult for the clinician to distinguish if these symptoms are due to an eating problem or a surgical consequence ${ }^{15}$. Patients may vomit in response to intolerable food or non-compliance to the dietary regimen imposed by bariatric surgery ${ }^{19}$. However, De Zwaan et al. have interviewed 59 patients 2 years after RYGB about a range of eating behaviors. Vomiting was reported by over $60 \%$ of the participants and self-induced vomiting with the goal of influencing shape and weight was admitted by $12 \%^{37}$. An intense fear of regaining weight or dissatisfaction with body image secondary to hanging skin, excessive preoccupation with weight and the use of restricting and purging behaviors have been referred by bariatric patients ${ }^{15,43}$. Furthermore, it was suggested that other postoperative undesirable events, such as "plugging" (problems with the small opening of the stomach becoming plugged with food) and dumping syndrome (intense discomfort after sweet ingestions accompanied by nausea, vomiting, bloating, cramping, diarrhea, dizziness, fatigue, weakness and sweating), may lead patients to engage in compensatory or restrictive behaviors. The reasons for this are the uncomfortable feeling of having overeaten or having consumed food that is difficult to tolerate after surgery ${ }^{15}$. Therefore, when examining postoperative 
eating patterns it is essential to investigate the motivation for them, especially because a postoperative eating disorder, such as bulimia nervosa can be easily confused with physical consequences of the surgical procedure.

All the postoperative inconsistent results make eating behavior a controversial area. Long-term research and standardized means of evaluation and diagnosis should be used in order to create clinical guidelines to ameliorate bariatric surgery follow-up and facilitate the early diagnosis of eating disorders after surgery.

\section{Mood disorders}

The majority of studies have reported a decrease in depression and depressive symptoms after bariatric surgery 24,27,48-56. Women seem to show a more marked decline than men ${ }^{57}$. Some studies have stated significantly reduced levels of depression symptoms 1,2 , and 10 years after bariatric surgery in comparison with the preoperative period ${ }^{5,58}$. Other findings indicate an initial improvement in the first years postoperatively followed by the reappearance or worsening of depressive symptoms which seem to be associated with weight regain or weight stabilization ${ }^{18}$. Studies have reported increased levels of depression after 5 years when compared to the first year following bariatric surgery ${ }^{59}$.

Considering anxiety symptoms, an overall improvement is observed after bariatric surgery $24,27,50-52$, however, it is smaller when compared to depression symptomatology ${ }^{58}$. On the contrary, other studies reveal no significant changes in anxiety measurements postoperatively $48,49,56$.

It has been suggested that a larger weight loss after surgery is associated with a more significant decrease in depressive and anxiety symptoms ${ }^{5,16,60}$. Additionally, a significant long-term relationship was found between depression and greater weight loss but the same did not happen for anxiety symptomology ${ }^{5}$. Moreover, some studies have demonstrated that bariatric patients may achieve normal scores of depression and anxiety following bariatric surgery ${ }^{19,51}$.

De Zwaan et al. have examined the course and prognostic significance of anxiety and depressive disorders in a sample of 107 bariatric patients using face-to-face interviews conducted prior to surgery and two times postoperatively (6-12 months and 24-36 months). They have reported that preoperative depressive disorder predicted depressive disorder 24-36 months after surgery whereas preoperative anxiety significantly predicted postoperative anxiety disorders at both follow-up time points. In addition, postoperative depressive disorder was significantly associated with less weight loss whilst postoperative anxiety disorder was not associated with the degree of weight loss at any follow-up time-point ${ }^{56}$.

Nevertheless, some studies do not report the expected improvement in depression and anxiety symptoms ${ }^{19}$. These inconsistent results may imply the presence of confounding factors. A prior trauma has been suggested as a possible candidate. Bariatric patients with traumatic background may present psychiatric complaints that are not fundamentally weight-related. Thus, traumatic histories might mediate the relationship between poorer postoperative weight loss and the maintenance of psychiatric symptoms ${ }^{14}$. Another implicated factor was the patient's expectations after surgery that may have a negative impact if the expected results are not obtained ${ }^{3}$. Furthermore, it may be difficult to cope with the persistence of pre-surgical problems or new negative life events which were attributed to obesity in the past ${ }^{18}$. Regarding psychiatric medication, some studies showed a decrease in their overall usage following bariatric surgery ${ }^{27}$. Although, one study has reported no cases of symptomatic depression postoperatively, $32 \%$ of patients were taking antidepressant medication to maintain the level of symptomatic control ${ }^{61}$. Analyzing antidepressant medication specifically, a study has stated that the number of patients being treated for anxiety and depression did not change during the 2 years period of follow-up ${ }^{58}$. Moreover, in a retrospective study, Cunningham et al. have studied the changes in the use of antidepressants medication after RYGB in a sample of 439 patients. These authors have observed that $23 \%$ of patients had an increase in their antidepressant use, $40 \%$ continued to require the same dose of antidepressant, $18 \%$ had a change in antidepressant medication and only $16 \%$ of patients had a decrease or discontinued the antidepressant therapy ${ }^{62}$. In addition, in a sample of 67 bariatric patients with high level of depressive symptomology was found that $30 \%$ used antidepressants preoperatively and $24 \%$ were on antidepressants after bariatric surgery ${ }^{55}$.

As the anatomy of the gastrointestinal tract is altered following bariatric surgery, drugs will not be absorbed as well. Beyond the absorption, distribution and elimination are pharmacokinetic processes we must consider. Each of these processes may be impacted by RYGB. With adjustable gastric banding the majority of these issues do not apply.

Roerig et al. have evaluated the changes in pharmacokinetics of the antidepressant sertraline after RYGB. There was a significantly smaller area under the plasma concentration/time curve in the postoperative group compared to the nonsurgical control group, which suggests an altered exposure to sertraline ${ }^{63}$. Despite the increasing numbers of bariatric surgeries performed, there is no accepted consensus regarding to antidepressant dosages after the different forms of bariatric surgery. Other aspect of concern is the choice of antidepressant taking into account the weight gain and the metabolic chances that may occur during the treatment with antidepressants ${ }^{64}$ Depressed patients are encouraged to receive pharmacologic treatment. Attempts should be made to place the patient on weight-neutral medications, avoiding medication such a tricyclic antidepressants or mirtazapine, which are known to cause weight gain ${ }^{65}$. For these reasons, postoperative monitoring of patients taking psychopharmacological medication is recommended. More knowledge needs to be acquired in the area of psychotropic medication pharmacokinetics in order to create clinical practice directives for the best care of bariatric patients ${ }^{66}$.

Three studies were found analyzing bipolar disorder (BD) and bariatric surgery. BD is considered in some bariatric programs as a definite contradiction to surgery while others consider BD with controlled symptom a possible contraindication ${ }^{67}$. Nevertheless, not a large number of studies were found that can confirm or refute these assumptions. Interestingly, one study has reported that patients with a lifetime mood disorder diagnosis had a significant lower weight loss than patients without a psychiatric disorder, however, after removing the patients with BD no significant differences were found in weight loss. Some explanations were elaborated, for example the impulsivity, which is characteristic of this disorder, might make difficult to adhere to behavioral modification imposed by the surgery. Furthermore, lithium, a medication which can be used in the treatment of $\mathrm{BD}$, has been shown to have a negative effect on memory and cognitive processing. Moreover, mood stabilizers are associated with an increased risk for several physical diseases, including obesity, dyslipidemia, diabetes mellitus ${ }^{68,69}$. On the contrary, another study has showed that patients with $\mathrm{BD}$ achieved positive weight loss outcomes at 12 months which were not significantly different from the patients with other psychiatric diagnosis or with no psychiatric disorder ${ }^{70}$. Additional research is needed regarding $\mathrm{BD}$ and bariatric surgery outcomes.

\section{Body image}

Body image dissatisfaction is more common in women and is associated with higher incidence of depression, low self-esteem and perfectionism ${ }^{13,54}$. A great psychological stress is observed due to stigmatization of obesity ${ }^{3}$.

Several studies demonstrated that body image impairment, attractiveness and lack of familiarity with the body improved after bariatric surgery16,19,71-73. Additionally, less body shape and weight concerns were reported ${ }^{74}$. However, most studies have only investigated changes in the first and second year after surgery ${ }^{54,72,75}$.

Although an overall improvement was found in body image after surgery, some studies have demonstrated poor results when 
compared to general population samples ${ }^{71}$ or non-obese populations ${ }^{54}$. Other authors have stated that body image-related concerns reached normal values postoperatively despite the fact that most patients continued to be overweight or obese $\mathrm{e}^{74}$.

Nevertheless, residual body image dissatisfaction due to increasing and/or sagging skin has been reported following weight loss surgery ${ }^{16,19,52,75,76}$. Recently, it has been established a correlation between the amount of excess skin and the degree of body image discomfort of bariatric patients ${ }^{76}$. Kinzl et al. have observed that $70 \%$ of obese patients who achieved a severe weight loss complained about flabby skin in their upper arms and thighs, abdominal flap and pendulous breasts. The authors have also stated that patients who were satisfied with their physical appearance lost less weight ${ }^{77}$. On the other hand, other studies have suggested that patients who had lost more weight were more satisfied with their image ${ }^{19}$.

The skin excess resulting from bariatric surgery is a common cause of functional and esthetical impairment which increases the desire and search for cosmetic surgery ${ }^{76,77}$. Pecori et al. have demonstrated in a small sample that patients who underwent aesthetic surgery had similar results in body image improvements in a 2-year follow-up when compared to patients who were only submitted to bariatric surgery. In addition, the authors have reported that individuals who sought aesthetic surgery had similar values of body uneasiness and dissatisfaction to those of severely obese patients72.

Therefore, bariatric surgeons must be advised to counsel their patients before surgery regarding body changes that they may experience postoperatively in order to prevent the psychological distress caused.

Inconsistent results were found relating body image and weight loss. Some studies have demonstrated that body image dissatisfaction did not correlate with weight loss ${ }^{54}$ or marginally correlated ${ }^{74}$, whereas, other authors have showed a more positive body image when the patient loses a greater amount of weight $53,71,78,79$. As a result, more studies are needed to elucidate the relationship between body image satisfaction and weight loss. Additionally, mediating variables that affect body image, such as self-esteem, appearance investment, social support and expectations of weight loss should be investigated.

Interestingly, one study was found in which body image satisfaction was compared between a night eaters group (NE) and control group (C) 5 months after RYBG. The two groups did not differ in weight loss or reductions in waist circumference. However, reported body image perceptions improved less in NE than C, the NE individuals saw themselves as heavier than they were ${ }^{47}$.

\section{Suicide}

Severely obese people may have an increased mortality by suicide as stated Mirabelli et al., in an Italian cohort study ${ }^{80}$. Other study which has evaluated the long-term mortality after bariatric surgery in a series of patient has reported a greater number of suicides in the surgical group comparing to the controls ${ }^{81}$. Tindle et al. have studied bariatric patients over a 10-years period and compared their suicide data to the U.S. and Pennsylvanian populations. They have reported an increase of suicides in the surgical group. The incidence of suicide in the surgical group was 6.6/10.000, which included 13.7/10.000 for men and 5.2/10.000 for women. These results were compared to the US population suicide rates $(2.4 / 10.000$ for men and $0.7 / 10.000$ for women). The majority of the deceased were Caucasian women. The reported average time to death was about 3 years after surgery $(10 \%$ in the first year, $29 \%$ in the second and $68 \%$ in the third). Furthermore, suicides were categorized by modes of death (drug overdose, gunshot wound, carbon monoxide poisoning and hanging ${ }^{82}$.

One study has been recently published comparing the reported suicide data after bariatric surgery with the Tindle et al. study and World Health Organization (WHO) data. Using 28 studies, they estimated a suicide rate after weight loss surgery of 4.1/10.000, which was significantly lower than the one reported by Tindle et al. ${ }^{82}$. Nevertheless, they report a four times higher probability of bariatric patients commit suicide when compared with WHO latest data (1.0/10.000). Regarding time frame, suicides occur between 18 months and 5 years after surgery ${ }^{83}$.

In this important problem of the suicide we must account with the multiple factors like the disinhibition and potential impulsivity secondary to changes in the alcohol absorption, the recurrence of medical comorbidities after bariatric surgery, the effect of malnutrition on the brain, the metabolic or pharmacokinetic changes that may affect the absorption of psychiatric medications, all of these can happen after bariatric surgery. Furthermore, we can not to have a superficial look with the association between suicide and the fact of have been submitted to a bariatric surgery. Recently, in a review, possible risk factors for increased suicide following bariatric surgery were addressed which included disappointment with the amount of weight loss, unresolved or recurrent medical conditions, and continued or recurrent physical mobility restrictions ${ }^{4}$. Unrealistic expectations about the outcomes of surgery, the distress with the limitations in the diet and the diverse care applied postoperatively, are potential factors attached to suicide risk in post-surgery period ${ }^{84}$. Patients need more intensive postoperative care, particularly medical, nutritional or psychotherapeutic care. A special aim of a multidisciplinary program must be to identify those patients who need specific interventions. Postoperative follow-up in multidisciplinary regimen (surgeon, endocrinologist, psychiatrist or psychologist and nutritionist) is of great importance ${ }^{85}$.

Although studies refer an increased rate of suicide, there is a significant variation in the characteristics of the studies and length of follow-up. Therefore, it is difficult to establish corrective comparisons between patients after bariatric surgery and the general population ${ }^{18}$.

\section{Substance use}

A prospective investigation of alcohol use disorders (AUD) before and after bariatric surgery found a significantly higher prevalence of AUD in the second postoperative year, overall, and specifically post-RYGB, compared to the years immediately before and following surgery ${ }^{86}$. Ertelt et al. have reported that a small percentage of the sample developed an AUD after gastric bypass, despite not having a previous dependence ${ }^{87}$. Moreover, one study has reported a higher frequency of alcohol abuse after RYGB than LAGB ${ }^{88}$.

Studies have reported that individuals submitted to bariatric surgery noticed that they become more intoxicated after consuming less alcohol and that there is a more rapid onset of intoxication effects comparing to the preoperative period ${ }^{87}$. Some studies showed that alcohol metabolism was significantly different between the postgastric bypass and control subjects ${ }^{89,90}$. Within minutes after consumption of a beverage containing a modest amount of alcohol, post-RYGB patients achieve disproportionately high blood alcohol concentration $s^{90}$. Considering alcohol pharmacokinetics, ethanol is metabolized primarily in the stomach by the gastric alcohol dehydrogenase enzyme. RYGB limits the first step of metabolism and, consequently, higher serum levels of ethanol are obtained ${ }^{36}$.

Conason et al. they have found a significant increase in alcohol use, recreational drug use and cigarette smoking 24 months after bariatric surgery ${ }^{88}$. In another study, 24 bariatric patients in an inpatient substance abuse treatment program were interviewed. Half of the sample stated that they had never experienced any alcohol or substance addiction prior to surgery ${ }^{91}$.

In a study conducted to determine opioid use following bariatric surgery in patients using opioids chronically for pain control prior to their surgery, $77 \%$ of them continued chronic opioid use in the year following surgery and the amount of opioid substance was greater postoperatively than preoperatively ${ }^{92}$. One possible explanation is that some patients likely had pain unresponsive to weight loss but potentially responsive to opioids. Multiple factors likely contribute to increasing chronic opioid use over time. Obese individuals demonstrate more pain sensitivity and lower pain detection thresholds than those who are not obese and altered pain processing persists after 
bariatric surgery. Contributing factors not unique to the bariatric surgery population include long-term opioid use possibly leading to tolerance, with need to take higher dosages to achieve equianalgesia and escalating dosages possibly increasing pain sensitivity, even when the initial cause has resolved ${ }^{9}$.

According to Weingarten et al., opioid consumption after bariatric surgery is greater among patients who are younger, men, and have been previously hospitalized because of psychiatric disorders. Additionally, they have reported that tobacco users tend to require more opioids and the current usage of psychotropic medications was not associated with greater opioid consumption or episodes of severe pain ${ }^{93}$.

A small amount of published data address this issue but it seems that despite detailed preoperative screening individuals can develop unforeseen substance addiction. More studies evaluating substance abuse postoperatively are necessary to assist in the understanding of this area.

\section{Conclusions}

Psychiatric disorders such as depressive, anxiety and binge eating disorders are prevalent among bariatric surgery candidates and an overall improvement in this psychopathology is observed after bariatric surgery. Nevertheless, depending on the specificity of the problem, the kind of comorbidity, the metabolic evolution and the motivation and treatment compliance, patients may or may not present psychopathological benefits postoperatively.

In addition it is important for clinicians to be aware of vomiting and other maladaptive behaviors because an eating disorder may be misdiagnosed.

Regarding body image, one increasing concern is the excessive skin that appears after substantial weight loss. A growing concern indicates a possible increased risk of suicide and substances abuse, especially alcohol, after bariatric surgery. However, suicide and use of substances of abuse remain relatively undeveloped topics since a small number of studies were found and different methodologies were used.

Further research is warranted for a more complete understanding of psychopatological areas in bariatric surgery patients. Furthermore, long-term studies are needed to elucidate the decline in improvements with time that is reported in some studies. This knowledge will allow a better preoperative selection, but above all, a more efficient follow-up.

\section{Conflict of interest}

None of the authors has conflict of interest.

\section{References}

1. Mango VL, Frishman WH. Physiologic, psychologic, and metabolic consequences of bariatric surgery. Cardiol Rev. 2006;14(5):232-7.

2. Sturm R, Hattori A. Morbid obesity rates continue to rise rapidly in the United States. Int J Obes (Lond). 2013;37(6):889-91.

3. van Hout G, van Heck G. Bariatric psychology, psychological aspects of weight loss surgery. Obes Facts.2009;2(1):10-5.

4. Muller A, Mitchell JE, Sondag C, de Zwaan M. Psychiatric aspects of bariatric surgery. Curr Psychiatry Rep. 2013;15(10):397.

5. Karlsson J, Taft C, Rydén A, Sjöström L, Sullivan M. Ten-year trends in health-related quality of life after surgical and conventional treatment for severe obesity: the SOS intervention study. Int J Obes (Lond). 2007;31(8):1248-61.

6. Buddeberg-Fischer B, Klaghofer R, Krug L, Buddeberg C, Müller MK, Schoeb O, et al. Physical and psychosocial outcome in morbidly obese patients with and without bariatric surgery: a 4 1/2-year follow-up. Obes Surg. 2006;16(3):321-30.

7. Ray EC, Nickels MW, Sayeed S, Sax HC. Predicting success after gastric bypass: the role of psychosocial and behavioral factors. Surgery. 2003;134(4):555-63; discussion 563-4.

8. Lee BO, Choi WJ, Sung NY, Lee SK, Lee CG, Kang JI. Incidence and risk factors for psychiatric comorbidity among people newly diagnosed with cancer based on Korean national registry data. Psychooncology. 2015.
9. Kang HJ, Kim SY, Bae KY, Kim SW, Shin IS, Yoon JS, et al. Comorbidity of depression with physical disorders: research and clinical implications. Chonnam Med J. 2015;51(1):8-18.

10. Lier HO, Biringer E, Hove O, Stubhaug B, Tangen T. Quality of life among patients undergoing bariatric surgery: associations with mental health- $A$ 1 year follow-up study of bariatric surgery patients. Health Qual Life Outcomes. 2011;9:79.

11. Lier HØ, Biringer E, Stubhaug B, Tangen T. Prevalence of psychiatric disorders before and 1 year after bariatric surgery: the role of shame in maintenance of psychiatric disorders in patients undergoing bariatric surgery. Nord J Psychiatry. 2013;67(2):89-96.

12. Kalarchian MA, Marcus MD, Levine MD, Courcoulas AP, Pilkonis PA, Ringham RM, et al. Psychiatric disorders among bariatric surgery candidates: relationship to obesity and functional health status. Am J Psychiatry. 2007;164(2):328-34.

13. Song A, Fernstrom MH. Nutritional and psychological considerations after bariatric surgery. Aesthet Surg J. 2008;28(2):195-9.

14. Rosik $\mathrm{CH}$. Psychiatric symptoms among prospective bariatric surgery patients: rates of prevalence and their relation to social desirability, pursuit of surgery, and follow-up attendance. Obes Surg. 2005;15(5):677-83.

15. Marino JM, Ertelt TW, Lancaster K, Steffen K, Peterson L, de Zwaan $M$, et al. The emergence of eating pathology after bariatric surgery: a rare outcome with important clinical implications. Int J Eat Disord. 2012;45(2):179-84.

16. Herpertz S, Kielmann R, Wolf AM, Langkafel M, Senf W, Hebebrand J. Does obesity surgery improve psychosocial functioning? A systematic review. Int J Obes Relat Metab Disord. 2003;27(11):1300-14.

17. Müller A, Mitchell JE, Sondag C, De Zwaan M. Psychiatric aspects of bariatric surgery topical collection on eating disorders. Curr Psychiatry Rep. 2013;15(10).

18. Kubik JF, Gill RS, Laffin M, Karmali S. The impact of bariatric surgery on psychological health. J Obes. 2013;2013:837989.

19. van Hout GC, Boekestein P, Fortuin FA, Pelle AJ, van Heck GL. Psychosocial functioning following bariatric surgery. Obes Surg. 2006;16(6):787-94.

20. van Hout G. Psychosocial effects of bariatric surgery. Acta Chir Belg. 2005;105(1):40-3.

21. Shiri S, Gurevich T, Feintuch U, Beglaibter N. Positive psychological impact of bariatric surgery. Obes Surg. 2007;17(5):663-8.

22. van Hout GC, Jakimowicz JJ, Fortuin FA, Pelle AJ, van Heck GL. Weight loss and eating behavior following vertical banded gastroplasty. Obes Surg. 2007;17(9):1226-34.

23. Scholtz S, Bidlake L, Morgan J, Fiennes A, El-Etar A, Lacey JH, et al. Long-term outcomes following laparoscopic adjustable gastric banding: postoperative psychological sequelae predict outcome at 5-year follow-up. Obes Surg. 2007;17(9):1220-5.

24. Sarwer DB, Wadden TA, Fabricatore AN. Psychosocial and behavioral aspects of bariatric surgery. Obes Res. 2005;13(4):639-48.

25. Birgegård A, Clinton D, Norring C. Diagnostic issues of binge eating in eating disorders. Eur Eat Disord Rev. 2013;21(3):175-83.

26. Wadden TA, Faulconbridge LF, Jones-Corneille LR, Sarwer DB, Fabricatore AN, Thomas JG, et al. Binge eating disorder and the outcome of bariatric surgery at one year: a prospective, observational study. Obesity (Silver Spring). 2011;19(6):1220-8.

27. Wolfe BL, Terry ML. Expectations and outcomes with gastric bypass surgery. Obes Surg. 2006;16(12):1622-9.

28. Kofman MD, Lent MR, Swencionis C. Maladaptive eating patterns, quality of life, and weight outcomes following gastric bypass: results of an Internet survey. Obesity (Silver Spring). 2010;18(10):1938-43.

29. Larsen JK, van Ramshorst B, Geenen R, Brand N, Stroebe W, van Doornen LJ. Binge eating and its relationship to outcome after laparoscopic adjustable gastric banding. Obes Surg. 2004;14(8):1111-7.

30. Herpertz S, Kielmann R, Wolf AM, Hebebrand J, Senf W. Do psychosocial variables predict weight loss or mental health after obesity surgery? A systematic review. Obes Res. 2004;12(10):1554-69.

31. Niego SH, Kofman MD, Weiss JJ, Geliebter A. Binge eating in the bariatric surgery population: a review of the literature. Int J Eat Disord. 2007;40(4):349-59.

32. Galioto R, Gunstad J, Heinberg LJ, Spitznagel MB. Adherence and weight loss outcomes in bariatric surgery: does cognitive function play a role? Obes Surg. 2013;23(10):1703-10. 
33. Guisado Macias JA, Vaz Leal FJ. Psychopathological differences between morbidly obese binge eaters and non-binge eaters after bariatric surgery. Eat Weight Disord. 2003;8(4):315-8.

34. Green AE, Dymek-Valentine M, Pytluk S, Le Grange D, Alverdy J. Psychosocial outcome of gastric bypass surgery for patients with and without binge eating. Obes Surg. 2004;14(7):975-85.

35. Israel A, Sebbag G, Fraser D, Levy I. Nutritional behavior as a predictor of early success after vertical gastroplasty. Obes Surg. 2005;15(1):88-94.

36. Odom J, Zalesin KC, Washington TL, Miller WW, Hakmeh B, Zaremba $\mathrm{DL}$, et al. Behavioral predictors of weight regain after bariatric surgery. Obes Surg. 2010;20(3):349-56.

37. de Zwaan M, Hilbert A, Swan-Kremeier L, Simonich H, Lancaster K, Howell LM, et al. Comprehensive interview assessment of eating behavior 18-35 months after gastric bypass surgery for morbid obesity. Surg Obes Relat Dis. 2010;6(1):79-85.

38. White MA, Kalarchian MA, Masheb RM, Marcus MD, Grilo CM. Loss of control over eating predicts outcomes in bariatric surgery patients: a prospective, 24-month follow-up study. J Clin Psychiatry. 2010;71(2):175-84.

39. Zunker C, Karr T, Saunders R, Mitchell JE. Eating behaviors post-bariatric surgery: a qualitative study of grazing. Obes Surg. 2012;22(8):1225-31.

40. Grimaldi D, Van Etten D. Psychosocial adjustments following weight loss surgery. J Psychosoc Nurs Ment Health Serv. 2010;48(3):24-9.

41. van Hout GC, Verschure SK, van Heck GL. Psychosocial predictors of success following bariatric surgery. Obes Surg. 2005;15(4):552-60.

42. Colles SL, Dixon JB, O’Brien PE. Hunger control and regular physical activity facilitate weight loss after laparoscopic adjustable gastric banding. Obes Surg. 2008;18(7):833-40.

43. Rusch MD, Andris D. Maladaptive eating patterns after weight-loss surgery. Nutr Clin Pract. 2007;22(1):41-9.

44. Leite Faria S, de Oliveira Kelly E, Pereira Faria O, Kiyomi Ito M. Snackeating patients experience lesser weight loss after Roux-en-Y gastric bypass surgery. Obes Surg. 2009;19(9):1293-6.

45. Colles SL, Dixon JB. Night eating syndrome: impact on bariatric surgery. Obes Surg. 2006;16(7):811-20.

46. Latner JD, Wetzler S, Goodman ER, Glinski J. Gastric bypass in a lowincome, inner-city population: eating disturbances and weight loss. Obes Res. 2004;12(6):956-61.

47. Morrow J, Gluck M, Lorence M, Flancbaum L, Geliebter A. Night eating status and influence on body weight, body image, hunger, and cortisol pre- and post- Roux-en-Y Gastric Bypass (RYGB) surgery. Eat Weight Disord. 2008;13(4):e96-9.

48. Assimakopoulos K, Karaivazoglou K, Panayiotopoulos S, Hyphantis T, Iconomou G, Kalfarentzos F. Bariatric surgery is associated with reduced depressive symptoms and better sexual function in obese female patients: a one-year follow-up study. Obes Surg. 2011;21(3):362-6.

49. Burgmer R, Petersen I, Burgmer M, de Zwaan M, Wolf AM, Herpertz S. Psychological outcome two years after restrictive bariatric surgery. Obes Surg. 2007;17(6):785-91.

50. Pataky Z, Carrard I, Golay A. Psychological factors and weight loss in bariatric surgery. Curr Opin Gastroenterol. 2011;27(2):167-73.

51. Thonney B, Pataky Z, Badel S, Bobbioni-Harsch E, Golay A. The relationship between weight loss and psychosocial functioning among bariatric surgery patients. Am J Surg. 2010;199(2):183-8.

52. Bocchieri LE, Meana M, Fisher BL. A review of psychosocial outcomes of surgery for morbid obesity. J Psychosom Res. 2002;52(3):155-65.

53. Dixon JB, O'Brien PE. Changes in comorbidities and improvements in quality of life after LAP-BAND placement. Am J Surg. 2002;184(6B):51S$54 \mathrm{~S}$.

54. Teufel M, Rieber N, Meile T, Giel KE, Sauer H, Hünnemeyer K, et al. Body image after sleeve gastrectomy: reduced dissatisfaction and increased dynamics. Obes Surg. 2012;22(8):1232-7.

55. Hayden MJ, Dixon JB, Dixon ME, Shea TL, O'Brien PE. Characterization of the improvement in depressive symptoms following bariatric surgery. Obes Surg. 2011;21(3):328-35.

56. de Zwaan M, Enderle J, Wagner S, Mühlhans B, Ditzen B, Gefeller O, et al. Anxiety and depression in bariatric surgery patients: a prospective, follow-up study using structured clinical interviews. J Affect Disord. 2011;133(1-2):61-8.

57. Mamplekou E, Komesidou V, Bissias Ch, Papakonstantinou A, Melissas J. Psychological condition and quality of life in patients with morbid obesity before and after surgical weight loss. Obes Surg. 2005;15(8):1177-84.

58. Andersen JR, Aasprang A, Bergsholm P, Sletteskog N, Våge V, Natvig GK. Anxiety and depression in association with morbid obesity: changes with improved physical health after duodenal switch. Health Qual Life Outcomes. 2010;8:52.

59. Strain GW, Saif T, Gagner M, Rossidis M, Dakin G, Pomp A. Crosssectional review of effects of laparoscopic sleeve gastrectomy at 1,3 , and 5 years. Surg Obes Relat Dis. 2011;7(6):714-9.

60. Thonney B, Pataky Z, Badel S, Bobbioni-Harsch E, Golay A. The relationship between weight loss and psychosocial functioning among bariatric surgery patients. Am J Surg. 2010;199(2):183-8.

61. Welch G, Wesolowski C, Zagarins S, Kuhn J, Romanelli J, Garb J, et al. Evaluation of clinical outcomes for gastric bypass surgery: results from a comprehensive follow-up study. Obes Surg. 2011;21(1):18-28.

62. Cunningham JL, Merrell CC, Sarr M, Somers KJ, McAlpine D, Reese $\mathrm{M}$, et al. Investigation of antidepressant medication usage after bariatric surgery. Obes Surg. 2012;22(4):530-5.

63. Roerig JL, Steffen K, Zimmerman C, Mitchell JE, Crosby RD, Cao L. Preliminary comparison of sertraline levels in postbariatric surgery patients versus matched nonsurgical cohort. Surg Obes Relat Dis. 2012;8(1):62-6.

64. Himmerich H, Minkwitz J, Kirkby KC. Weight gain and metabolic changes during treatment with antipsychotics and antidepressants. Endocr Metab Immune Disord Drug Targets. 2015.

65. Sarwer DB, Cohn NI, Gibbons LM, Magee L, Crerand CE, Raper SE, et al. Psychiatric diagnoses and psychiatric treatment among bariatric surgery candidates. Obes Surg. 2004;14(9):1148-56.

66. Geraldo MS, Fonseca FL, Gouveia MR, Feder D. The use of drugs in patients who have undergone bariatric surgery. Int J Gen Med. 2014;7:219-24.

67. Ahmed AT, Blair TR, McIntyre RS. Surgical treatment of morbid obesity among patients with bipolar disorder: a research agenda. Adv Ther. 2011;28(5):389-400.

68. Semanscin-Doerr DA, Windover A, Ashton K, Heinberg LJ. Mood disorders in laparoscopic sleeve gastrectomy patients: does it affect early weight loss? Surg Obes Relat Dis. 2010;6(2):191-6.

69. Correll CU, Detraux J, De Lepeleire J, De Hert M. Effects of antipsychotics, antidepressants and mood stabilizers on risk for physical diseases in people with schizophrenia, depression and bipolar disorder. World Psychiatry. 2015;14(2):119-36.

70. Steinmann WC, Suttmoeller K, Chitima-Matsiga R, Nagam N, Suttmoeller NR, Halstenson NA. Bariatric surgery: 1-year weight loss outcomes in patients with bipolar and other psychiatric disorders. Obes Surg. 2011;21(9):1323-9.

71. van Hout GC, Fortuin FA, Pelle AJ, van Heck GL. Psychosocial functioning, personality, and body image following vertical banded gastroplasty. Obes Surg. 2008;18(1):115-20.

72. Pecori L, Serra Cervetti GG, Marinari GM, Migliori F, Adami GF. Attitudes of morbidly obese patients to weight loss and body image following bariatric surgery and body contouring. Obes Surg. 2007;17(1):68-73.

73. Madan AK, Beech BM, Tichansky DS. Body esteem improves after bariatric surgery. Surg Innov. 2008;15(1):32-7.

74. Hrabosky JI, Masheb RM, White MA, Rothschild BS, Burke-Martindale $\mathrm{CH}$, Grilo CM. A prospective study of body dissatisfaction and concerns in extremely obese gastric bypass patients: 6- and 12-month postoperative outcomes. Obes Surg. 2006;16(12):1615-21.

75. Sarwer DB, Fabricatore AN. Psychiatric considerations of the massive weight loss patient. Clin Plast Surg. 2008;35(1):1-10.

76. Staalesen T, Fagevik Olsén M, Elander A. Experience of excess skin and desire for body contouring surgery in post-bariatric patients. Obes Surg. 2013;23(10):1632-44.

77. Kinzl JF, Traweger C, Trefalt E, Biebl W. Psychosocial consequences of weight loss following gastric banding for morbid obesity. Obes Surg. 2003;13(1):105-10.

78. Guisado JA, Vaz FJ, Alarcón J, López-Ibor JJ Jr, Rubio MA, Gaite L. Psychopathological status and interpersonal functioning following weight loss in morbidly obese patients undergoing bariatric surgery. Obes Surg. 2002;12(6):835-40

79. Zijlstra H, Larsen JK, van Ramshorst B, Geenen R. The association between weight loss and self-regulation cognitions before and after laparoscopic adjustable gastric banding for obesity: a longitudinal study. Surgery. 2006;139(3):334-9.

80. Mirabelli D, Petroni ML, Ferrante D, Merletti F. Risk of suicide and bariatric surgery. Am J Med. 2011;124(8):e17.

81. Adams TD, Gress RE, Smith SC, Halverson RC, Simper SC, Rosamond $\mathrm{WD}$, et al. Long-term mortality after gastric bypass surgery. $\mathrm{N}$ Engl J Med. 2007;357(8):753-61. 
82. Tindle HA, Omalu B, Courcoulas A, Marcus M, Hammers J, Kuller LH. Risk of suicide after long-term follow-up from bariatric surgery. Am J Med. 2010;123(11):1036-42.

83. Peterhänsel C, Petroff D, Klinitzke G, Kersting A, Wagner B. Risk of completed suicide after bariatric surgery: a systematic review. Obes Rev. 2013;14(5):369-82.

84. Diniz Mde F, Moura LD, Kelles SM, Diniz MT. Long-term mortality of patients submitted to Roux-en-Y gastric bypass in Public Health System: high prevalence of alcoholic cirrhosis and suicides. Arq Bras Cir Dig. 2013;26 Suppl 1:53-6.

85. Pajecki D, Mancini MC, Halpern A, Zilberstein B, Garrido AB Jr, Cecconello I. Multidisciplinary approach to morbidly obese patients undergoing surgical treatment by adjustable gastric banding. Rev Col Bras Cir. 2010;37(5):328-32.

86. King WC, Chen JY, Mitchell JE, Kalarchian MA, Steffen KJ, Engel SG, et al. Prevalence of alcohol use disorders before and after bariatric surgery. JAMA. 2012;307(23):2516-25.

87. Ertelt TW, Mitchell JE, Lancaster K, Crosby RD, Steffen KJ, Marino JM. Alcohol abuse and dependence before and after bariatric surgery: a review of the literature and report of a new data set. Surg Obes Relat Dis. 2008;4(5):647-50.

88. Conason A, Teixeira J, Hsu CH, Puma L, Knafo D, Geliebter A. Substance use following bariatric weight loss surgery. JAMA Surg. 2013;148(2):145-50

89. Hagedorn JC, Encarnacion B, Brat GA, Morton JM. Does gastric bypass alter alcohol metabolism? Surg Obes Relat Dis. 2007;3(5):543-8.

90. Steffen KJ, Engel SG, Pollert GA, Li C, Mitchell JE. Blood alcohol concentrations rise rapidly and dramatically after Roux-en-Y gastric bypass. Surg Obes Relat Dis. 2013;9(3):470-3.

91. Ivezaj V, Saules KK, Wiedemann AA. "I didn't see this coming.": why are postbariatric patients in substance abuse treatment? Patients perceptions of etiology and future recommendations. Obes Surg. 2012;22(8):1308-14.

92. Raebel MA, Newcomer SR, Reifler LM, Boudreau D, Elliott TE, DeBar $\mathrm{L}$, et al. Chronic use of opioid medications before and after bariatric surgery. JAMA. 2013;310(13):1369-76.

93. Weingarten TN, Sprung J, Flores A, Baena AM, Schroeder DR, Warner DO. Opioid requirements after laparoscopic bariatric surgery. Obes Surg. 2011;21(9):1407-12. 